MPI-Ph/93-58

SSCL-Preprint-497

August 1993

\title{
Infrared Fixed Point Solution for the Top Quark Mass and Unification of Couplings in the MSSM
}

\author{
W. A. Bardeen ${ }^{a}$, M. Carena ${ }^{b}$, \\ S. Pokorski ${ }^{b *}$ and C. E. M. Wagner ${ }^{b}$ \\ (a) Theoretical Physics, SSC Laboratory, \\ 2550 Beckleymeade Ave., Dallas, Texas 75237-3946, U.S.A. \\ ${ }^{(b)}$ Max-Planck-Institut für Physik, Werner-Heisenberg-Institut \\ Föhringer Ring 6, D-80805 München, Germany.
}

\begin{abstract}
We analyze the implications of the infrared quasi fixed point solution for the top quark mass in the Minimal Supersymmetric Standard Model. This solution could explain in a natural way the relatively large value of the top quark mass and, if confirmed experimentally, may be suggestive of the onset of nonperturbative physics at very high energy scales. In the framework of grand unification, the expected bottom quark - tau lepton Yukawa coupling unification is very sensitive to the fixed point structure of the top quark mass. For the presently allowed values of the electroweak parameters and the bottom quark mass, the Yukawa coupling unification implies that the top quark mass must be within ten percent of its fixed point values.
\end{abstract}

\footnotetext{
${ }^{*}$ On leave from the Institute of Theoretical Physics, Warsaw University
} 
In the present expectation of a very heavy top quark, we witness a revival of interest in the infrared quasi fixed point predictions for the top quark mass. The idea is that the top quark mass may be completely determined by the low energy fixed point structure of the Renormalization Group ( $R G$ ) equations independent of the precise symmetry conditions at a high mass scale. For several reasons, this idea is very appealing. The infrared stable fixed point structure of the Standard Model was first analysed by Pendleton and Ross [1]. Their analysis focussed on an exact fixed point relationship between the top quark Yukawa coupling and the QCD coupling which requires a smooth running of the couplings to infinite energy or implies analytic relations between the couplings. This approach is closely related to the coupling reduction methods advocated by Zimmermann et al. [2]. At difference with the Pendleton-Ross fixed point, the quasi fixed point structure of C. T. Hill [3] results from a strong focussing of the running of the top quark Yukawa at low energies for sufficiently strong Yukawa couplings at a finite high energy scale. This behavior reflects the existence of Landau poles or nonanalytic relations between the couplings in the RG evolution somewhat above the high energy scale, where new physics is expected to control the actual values of the couplings. A wide range of couplings at the high energy scale will fall within the domain of attraction of the quasi fixed point and evolve to a sharply defined fixed point value for the top quark mass. The fixed point structure of the Standard Model provides a natural explanation of larger values of the top quark mass.

In the Minimal Supersymmetric Standard Model (MSSM), a similar quasi fixed point behavior of the renormalization group solutions is present [4]. In fact, for a given value of the strong gauge coupling $\alpha_{3}\left(M_{Z}\right)$, one predicts

$$
M_{t} \simeq A \sin \beta
$$

where $M_{t}$ is the physical top quark mass, the angle $\beta$ is defined so that $\tan \beta$ is the ratio of the two Higgs vacuum expectation values and $A \simeq 190-210 \mathrm{GeV}$ for $\alpha_{3}\left(M_{Z}\right)=0.11-0.13$ [5] - [7. It is worth stressing that the above prediction is obtained for a range of high energy values of $Y_{t}=h_{t}^{2} / 4 \pi, h_{t}$ being the top quark Yukawa coupling, such that $Y_{t}$ can reach its perturbativity limit $Y_{t} \simeq \mathcal{O}(1)$ at some scale $M_{X}=10^{14}-10^{19} \mathrm{GeV}$ (see Fig. 1). One can therefore envision the following scenarios, which would lead to the infrared quasi fixed point prediction for $M_{t}$ :

i) The onset of non-perturbative physics at scales below or of the order $M_{G U T}=\mathcal{O}\left(10^{16}\right.$ $\mathrm{GeV}$ ), at which the unification of gauge couplings may take place. This is what happens, 
for example, in the supersymmetric extension of the so called top condensate models 《4. In this case, the perturbative analysis of grand unified scenarios would be invalidated and one should make an analysis of nonperturbative effects before studying the precise unification conditions.

ii) Perturbative grand unification, with a value of $0.1 \leq Y_{t} \leq 1$ at the grand unification scale, followed by the onset of nonperturbative physics for scales of the order of $M_{G U T}$.

iii) Perturbative theory up to scales of the order of $M_{P L}$, but with large $Y_{t}$, close to its perturbativity limit, and with the possibility of new physics at scales $\mu \geq M_{G U T}$.

In summary, an infrared quasi fixed point value for $M_{t}$, if confirmed experimentally, would either be strongly suggestive of non-perturbative physics at very high energy scales, or would call for understanding the relatively large values, of order one, of the top quark Yukawa coupling at the high energy scales within the perturbative scenario.

Going further in the aim of computing the values of the other fermion masses measured experimentally, it is natural to think about the possibility of viewing them in the framework of grand unified theories. In this respect, bottom-tau Yukawa coupling unification appears naturally in many grand unified schemes [8]- [10]. Most interesting is the fact that to achieve bottom-tau Yukawa unification, $h_{b}\left(M_{G U T}\right)=h_{\tau}\left(M_{G U T}\right)$, large values of the top quark Yukawa coupling are necessary in order to compensate for the effects of the strong interaction renormalization. Indeed, most recently, it has been observed that one is driven close to the infrared quasi fixed point by the bottom - tau Yukawa unification requirement [5], [7]. This property is strongly dependent on the exact values of the strong gauge coupling as well as on the physical bottom quark mass being in the range of experimentally allowed values $M_{b}=4.9 \pm 0.3 \mathrm{GeV}$ [11]. If, for example, the physical bottom mass value were $M_{b} \leq 3 \mathrm{GeV}$, perturbative unification of bottom and tau Yukawa couplings would not be possible for $\alpha_{3}\left(M_{Z}\right)=0.11-0.13$, since even a top quark Yukawa coupling at the edge of the validity of the perturbative expansion would not be strong enough to contravene the strong gauge coupling renormalization of the bottom mass. In fact, for a given bottom mass, one can define an upper bound on the strong gauge coupling for which bottom-tau Yukawa unification becomes possible [7]. Surprisingly enough, for experimentally allowed values of $M_{b}$, this upper bound on $\alpha_{3}\left(M_{Z}\right)$ lies within the range predicted from LEP measurements. In addition, the experimental upper bound on $M_{b}$ is also quite important to account for the infrared fixed point behaviour of the top quark mass. For larger values of the bottom quark mass, $M_{b} \geq 6 \mathrm{GeV}$, there would be no necessity of a strong renormalization effect from the 
top quark Yukawa coupling.

The above is quite intriguing, since it implies that just for the experimentally acceptable values of $M_{b}$ and $\alpha_{3}\left(M_{Z}\right)$, bottom-tau Yukawa unification yields large values -of order oneof the top Yukawa coupling at the grand unification scale, which, however, remain in the range of validity of perturbation theory. This is a highly nontrivial property of the Minimal Supersymmetric Standard Model and, as we explained above, it implies that the top quark mass is strongly focussed to its infrared quasi fixed point value. Thus, in this case, the observed top quark mass will be insensitive to the actual value of the top quark Yukawa coupling at high energies. It is precisely the bottom-tau Yukawa unification condition which would be uniquely sensitive to the high energy Yukawa coupling of the top quark.

In the following, we extend and quantify the previous discussion. In particular, we address the issue of the proximity to the infrared quasi fixed point prediction for $M_{t}$ when requiring $h_{b}\left(M_{G U T}\right)=h_{\tau}\left(M_{G U T}\right)$. In Figs. 2.a - 2.d, we consider the two loop renormalization group running of the bottom quark and tau lepton Yukawa couplings, $Y_{b}=h_{b}^{2} / 4 \pi$ and $Y_{\tau}=h_{\tau}^{2} / 4 \pi$, respectively, for two representative values of $\alpha_{3}\left(M_{Z}\right)=0.11,0.125$, and the physical bottom quark mass $M_{b}=4.9,5.2 \mathrm{GeV}$, to analyze under which conditions the unification of these two Yukawa couplings is possible. The larger (smaller) the value of $\alpha_{3}\left(M_{Z}\right)\left(M_{b}\right)$ the larger is the value of $h_{t}$ necessary to achieve unification of $h_{b}$ and $h_{\tau}$ in the range, say $10^{15}-10^{19} \mathrm{GeV}$. As we discussed above, there is an obvious reason for this: Large $h_{t}$ is needed to partially cancel the strong interaction renormalization of $M_{b}, h_{t}$ being necessarily strong at scales of order $M_{G U T}$ for $M_{b}=4.9 \pm 0.3 \mathrm{GeV}$ and large values of $\alpha_{3}\left(M_{Z}\right) \geq 0.115$. At the same time, for large values of the top quark Yukawa coupling at the grand unification scale, $h_{t}\left(M_{G U T}\right)$, for which the top mass is close to its quasi infrared fixed point value, the $h_{b}-h_{\tau}$ unification scale becomes extremely sensitive to the actual top quark mass value. Changing $M_{t}$ by 1 $3 \mathrm{GeV}$ (for fixed $\tan \beta$ ) can change the bottom - tau unification scale by several orders of magnitude. This implies, as shown in Fig. 2, that for $\alpha_{3}\left(M_{Z}\right)=0.125$, unification of $h_{b}$ and $h_{\tau}$ in the range $M_{G U T}=10^{15}-10^{19} \mathrm{GeV}$ only holds for very restrictive values of the running top quark mass, $m_{t}$. [7 For instance, $\mathrm{a} \tan \beta=3.5$, a physical bottom mass $M_{b}=4.9 \mathrm{GeV}$ and $\alpha_{3}\left(M_{Z}\right)=0.125$ implies $m_{t} \simeq 190 \pm 3 \mathrm{GeV}$. Similarly, values of $\alpha_{3}\left(M_{Z}\right)=0.12(0.115)$ imply $m_{t} \simeq 186 \pm 3 \mathrm{GeV}(182 \pm 3 \mathrm{GeV})$. Moreover, as can be clearly seen in Fig. 2, for these values of $\alpha_{3}\left(M_{Z}\right)$ the predicted top quark mass is quite insensitive to the inclusion of small

\footnotetext{
${ }^{1}$ Once the QCD corrections are included, the running top quark mass used above is about $6 \%$ smaller than the physical pole mass [7].
} 
threshold corrections, of the order of $10 \%$, to the bottom-tau Yukawa coupling unification condition. On the contrary, for smaller values of $\alpha_{3}\left(M_{Z}\right) \leq 0.110$, unification of $h_{b}$ and $h_{\tau}$ may, in principle, be achieved for a wide range of smaller values of $m_{t}$. For example, for $\alpha_{3}\left(M_{Z}\right)=0.105, \tan \beta=3.5$ and considering $M_{b}=5.2 \mathrm{GeV}$, bottom - tau Yukawa unification is possible for values of $m_{t} \simeq 125-160 \mathrm{GeV}$. However, as we shall show below, for a top quark mass $M_{t}>110 \mathrm{GeV}$, these relatively low values of the strong gauge coupling, $\alpha_{3}\left(M_{Z}\right) \leq 0.110$, can not be self consistently achieved if unification of gauge couplings is also required for the MSSM. Similar features are obtained for the whole low and moderate $\tan \beta$ regime.

Next, we study in more detail how close are we driven to the infrared fixed point prediction for $M_{t}$ by the requirement of $h_{b}-h_{\tau}$ unification, as a function of the strong gauge coupling and for several values of the bottom quark mass and $\tan \beta$. The $h_{b}-h_{\tau}$ unification scale is defined by the running of the gauge couplings. The results are shown in Fig. 3.a - 3.c. We see that, for values of the strong gauge coupling $\alpha_{3}\left(M_{Z}\right) \geq 0.115$, and for $M_{b} \leq 5.2$ $\mathrm{GeV}$, the top quark mass is within a ten percent of its infrared quasi fixed point value. If, instead, the strong gauge coupling were $\alpha_{3}\left(M_{Z}\right)<0.110$, the top quark mass could be far away from its infrared quasi fixed point value. Concerning possible threshold corrections to the unification of both Yukawa couplings, it is interesting to remark that a relaxation in the unification condition of order $10 \%, h_{b}\left(M_{G U T}\right) / h_{\tau}\left(M_{G U T}\right)=0.9$, for a bottom mass $M_{b}=4.9$ $\mathrm{GeV}$ gives approximately the same behaviour as if we consider exact $h_{b}-h_{\tau}$ unification but with $M_{b}=5.2 \mathrm{GeV}$.

It is important to observe that for smaller values of $M_{b}$ and larger values of $\alpha_{3}\left(M_{Z}\right)$ the top quark Yukawa coupling required for $h_{b}-h_{\tau}$ unification may become too large. For a consistent perturbative treatment of the theory we require $Y_{t}\left(M_{G U T}\right)=h_{t}^{2}\left(M_{G U T}\right) / 4 \pi \leq 1$, which implies that the two loop contribution to the renormalization group evolution of $h_{t}$ is less than a $30 \%$ of the one loop one. As a matter of fact, observe that in Fig. 3. the curves for $M_{b}=4.7 \mathrm{GeV}$ and $M_{b}=4.9 \mathrm{GeV}$ do not continue up to $\alpha_{3}\left(M_{Z}\right)=0.13$, since the top quark Yukawa coupling at $M_{G U T}$ would then become too large to be consistent with a perturbative analysis [7].

Considering the constraints coming from the gauge coupling unification [12] - [16], predictions for $\sin ^{2} \theta_{W}\left(M_{Z}\right)$ are derived as a function of the strong gauge coupling $\alpha_{3}\left(M_{Z}\right)$. 
Indeed, the unification condition implies the following numerical correlation

$$
\sin ^{2} \theta_{W}\left(M_{Z}\right)=0.2324-0.25 \times\left(\alpha_{3}\left(M_{Z}\right)-0.123\right) \pm 0.0025,
$$

where the central value corresponds to an effective supersymmetric threshold scale [6], [7] $T_{S U S Y}=M_{Z}$ and the error \pm 0.0025 is the estimated uncertainty in the prediction arising from possible supersymmetric threshold corrections (corresponding to varying $T_{S U S Y}$ from $15 \mathrm{GeV}$ to $1 \mathrm{TeV}$ ) and including possible effects from threshold corrections at the unification scale as well as from higher dimensional operators. On the other hand, $\sin ^{2} \theta_{W}\left(M_{Z}\right)$ is given by the electroweak parameters $G_{F}, M_{Z}, \alpha_{e m}$ as a function of $M_{t}$ (at the one - loop level) by the formula [6]:

$$
\sin ^{2} \theta_{W}\left(M_{Z}\right)=0.2324-10^{-7} \times G e V^{-2} \times\left(M_{t}^{2}-(138 G e V)^{2}\right) \pm 0.0003
$$

Therefore, the predictions from the gauge coupling unification agree with experimental data provided

$$
M_{t}^{2}=(138 G e V)^{2}+10^{7} \times G e V^{2} \times 0.25 \times\left(\alpha_{3}\left(M_{Z}\right)-0.123 \pm 0.01\right)
$$

The above $M_{t}-\alpha_{3}\left(M_{Z}\right)$ correlation defines a band, whose upper bound is $\alpha_{3}^{u}\left(M_{Z}\right) \geq 0.13$. Thus, although this upper bound does not appear explicitly in Fig. 3., Eq (四) implies that the region in $\alpha_{3}\left(M_{Z}\right)$ to the right of the dash-long dashed curve is the allowed one. Moreover, in Fig. 3 we observe the intersection of that allowed region with the $M_{t}-\alpha_{3}\left(M_{Z}\right)$ curves which follow from the $h_{b}-h_{\tau}$ unification condition. In fact, for $M_{b}$ in the range $(4.9 \pm 0.3)$ $\mathrm{GeV}$, the $h_{b}-h_{\tau}$ unification and the gauge coupling unification condition together with the quadratic dependence on $M_{t}$ of $\sin ^{2} \theta_{W}\left(M_{Z}\right)$ (both within the discussed uncertainties) are compatible with each other only within a restricted range of $\alpha_{3}\left(M_{Z}\right)$ and $M_{t}$, and, moreover, push $M_{t}$ very close to its quasi infrared fixed point values. Due to the correlation between $\sin ^{2} \theta_{W}\left(M_{Z}\right)$ and $M_{t}$, the effect becomes more dramatic for larger values of $\tan \beta$, for which $\sin \beta \simeq 1$, than for values of $\tan \beta$ close to one. In general, for $\tan \beta \geq 1$, the strong gauge coupling takes values $\alpha_{3}\left(M_{Z}\right) \geq 0.112$ and $M_{t}$ is at most a $10 \%$ lower than its infrared quasi fixed point prediction. Indeed, as may be observed from Fig. 3, the above lower bound on $\alpha_{3}\left(M_{Z}\right)$ may only be reached for $\tan \beta \simeq 1$. For larger values of $\tan \beta$, the lower bound on $\alpha_{3}\left(M_{Z}\right)$ increases together with the top quark mass, which has then a stronger convergence to its infrared fixed point behavior.

It is important to remark that the above study is performed in the region of moderate values of $\tan \beta \leq 30$. In this region, the top Yukawa coupling at the grand unification scale 
depends only weakly on the exact supersymmetric spectrum but strongly on the effective supersymmetric threshold scale $T_{S U S Y}$ [7] which determines the low energy values of the strong gauge coupling coming from the requirement of gauge coupling unification [6]. The present figures were obtained for $T_{S U S Y}=M_{Z}$, taking the squark masses to be equal to the $Z$ boson mass. If, while keeping $T_{S U S Y}$ fixed, the squark masses are increased, in the range $M_{Z}-1 \mathrm{TeV}$, the top quark mass may increase in a few $\mathrm{GeV}$ but without changing the physical picture [0].

For larger values of $\tan \beta$, for which the bottom Yukawa coupling becomes sufficiently strong to be able to partially cancel the strong gauge coupling renormalization effects in its own running, the $h_{b}-h_{\tau}$ unification condition no longer requires the existence of a large top Yukawa coupling [7]. Therefore, the predictions for $M_{t}$ are no longer strongly constrained to be close to its infrared quasi fixed point values. As shown in Fig. 3.d, already for values of $M_{b}$ of the order of its experimental upper bound, large deviations from the infrared quasi fixed point predictions for $M_{t}$ are observed, for a wide range of experimentally allowed values for $\alpha_{3}\left(M_{Z}\right)$. In addition, for very large values of $\tan \beta$, large corrections to the running bottom quark mass may be present [17],[18], and additional symmetries may be required to cancel them in a natural way. Such symmetries may, however, be in conflict with the radiative breaking of $S U(2)_{L} \times U(1)_{Y}$ [19]. The loss of stability for the top quark mass values in the large $\tan \beta$ regime is also reflected in a stronger sensitivity to the exact supersymmetric spectrum. Furthermore, in Fig. 3.d. it is to observe that for $\tan \beta=50$ the condition of unification of the three Yukawa couplings, $h_{t}\left(M_{G U T}\right)=h_{b}\left(M_{G U T}\right)=h_{\tau}\left(M_{G U T}\right)$ (dot-long dashed line) determines a large value of the top quark mass which, however differs from the infrared fixed point behavior. In this case, the value of the Yukawa couplings at the unification scale is of the order of the unification gauge coupling. In addition, for lower values of the bottom quark mass the predictions for $M_{t}$ are outside the range allowed by the gauge coupling unification condition. To achieve unification of the three Yukawa couplings inducing as well values of the top quark mass close to its infrared fixed point, a larger value of $\tan \beta$ is required.

Finally, concerning the present experimental limits on the top quark mass it follows that, for a Higgs mass $m_{h} \simeq 100 \mathrm{GeV}$, the direct experimental determination of the Weinberg angle from LEP yields $M_{t} \simeq 150 \pm 40 \mathrm{GeV}$ at the $95 \%$ confidence level [20]. The same result would be obtained in the Minimal Supersymmetric Standard Model, if all supersymmetric particle 
masses $M_{S U S Y} \gg M_{Z}$. However, as has been recently pointed out, this result could be modified in case there were light supersymmetric particles, with masses close to their present experimental bound [21], 22]. In the heavy MSSM scenario, with no light supersymmetric particles, the rather large prediction for the top quark mass coming from the most recent experimental measurements allows the accomodation of the above discussed scenario for a reasonable range of values of $\tan \beta$ at the two sigma level. The light minimal supersymmetric scenario may even improve the agreement, depending on which supersymmetric particles become light.

In conclusion, in the Minimal Supersymmetric Standard Model, for any given value of the strong gauge coupling $\alpha_{3}\left(M_{Z}\right)$, the infrared quasi fixed point solution naturally provides predictions of a large top quark mass as a function of $\tan \beta$. We have shown that such values of the top quark mass may also be naturally obtained within the context of grand unified scenarios with gauge and bottom-tau Yukawa coupling unification. Indeed, for small and moderate values of $\tan \beta$ and a physical bottom mass $M_{b}=4.9 \pm 0.3 \mathrm{GeV}$, the top quark mass necessary to achieve unification of gauge and bottom-tau Yukawa couplings is within a ten percent of the infrared quasi fixed point predictions for this quantity. This result is not modified under the inclusion of low and high energy threshold corrections to the gauge couplings. In addition, we have shown that, whenever the top quark mass is close to its infrared quasi fixed point value, the predictions for this quantity become stable under variations of the bottom - tau Yukawa unification scale as well as under small high energy threshold corrections on the bottom and tau Yukawa couplings. Finally, it is worth emphasizing that the experimental confirmation of the infrared quasi fixed point solution will require not only the knowledge of the top quark mass, but of $\tan \beta$ as well, an additional information which may come, for example, from the Higgs sector of the theory. If confirmed experimentally, the infrared quasi fixed point solution will demand for understanding the large values of the top quark Yukawa coupling at scales $Q=\mathcal{O}\left(M_{G U T}\right)$, either within the perturbative scenario or from nonperturbative physics at these high energy scales.

Acknowledgements: W.B. would like to thank members of the Max Planck Institute for Physics for their hospitality and support for this research. Useful discussions with M. Olechowski are gratefully acknowledged. S. P. is partially supported by the Polish Committee for Scientific Research. 


\section{FIGURE CAPTIONS}

Fig. 1. Top quark mass renormalization group running, as a function of $x=\ln \left(Q / M_{Z}\right)^{2}$, for different boundary conditions of the top quark Yukawa coupling at the scale $M_{X}=10^{16}$ $\mathrm{GeV}, \alpha_{3}\left(M_{Z}\right)=0.12$ and $\tan \beta=5$.

Fig. 2. Bottom quark (solid line) and Tau (dashed line) Yukawa coupling renormalization group running as a function of the renormalization group scale $Q$, for $\tan \beta=3.5$ and for a) a bottom quark mass $M_{b}=4.9 \mathrm{GeV}, \alpha_{3}\left(M_{Z}\right)=0.11$ and different values of the running top quark mass, which, starting from below read $m_{t}[\mathrm{GeV}]=134,154,169,174,176$, 177, 179, 180; b) $M_{b}=5.2 \mathrm{GeV}, \alpha_{3}\left(M_{Z}\right)=0.11$ and $m_{t}[G e V]=134,154,161,170,173$, $176,177,179,180$; c) $M_{b}=4.9 \mathrm{GeV}, \alpha_{3}\left(M_{Z}\right)=0.125$ and $m_{t}[G e V]=134,154,174,184$, $187,188,189,191,192 ;$ d) $M_{b}=5.2 \mathrm{GeV}, \alpha_{3}\left(M_{Z}\right)=0.125$ and $m_{t}[G e V]=134,154,174$, 184, 187, 188, 190. The tau Yukawa coupling being approximately the same for all the above values of $m_{t}$.

Fig. 3. Comparison of the infrared quasi fixed point top quark mass predictions (solid line) with the top quark mass necessary to achieve bottom - tau Yukawa coupling unification as a function of the strong gauge coupling $\alpha_{3}\left(M_{Z}\right)$, for $M_{b}=5.2 \mathrm{GeV}$ (dot-dashed line), $M_{b}=4.9 \mathrm{GeV}$ (long dashed line) and $M_{b}=4.7 \mathrm{GeV}$ (dashed line), and for a) $\tan \beta=1 ; \mathrm{b}$ ) $\tan \beta=2$; c) $\tan \beta=5$; d) $\tan \beta=50$, where the dot-long dashed line represents the values at which the three Yukawa couplings unify. Also shown in the figure is the band predicted from the condition of gauge coupling unification and the experimental correlation between $M_{t}$ and $\sin ^{2} \theta_{W}\left(M_{Z}\right)$, which extends to the right of the dash-long dashed line. 


\section{References}

[1] B. Pendleton and G. G. Ross, Phys. Lett. 98B (1981) 291.

[2] W. Zimmermann, Comm. Math. Phys. 97 (1985) 211;

J. Kubo, K. Sibold and W. Zimmermann, Phys. Lett. B200 (1989) 191.

[3] C. T. Hill, Phys. Rev. D24 (1981) 691;

C. T. Hill, C. N. Leung and S. Rao, Nucl. Phys. B262 (1985) 517.

[4] M. Carena, T.E. Clark, C.E.M. Wagner, W.A. Bardeen and K. Sasaki, Nucl. Phys. B369 (1992) 33.

[5] V. Barger, M.S. Berger and P. Ohmann, Phys. Rev. D 47 (1993) 1093; V. Barger, M.S. Berger, P. Ohmann and R.J.N. Phillips, U. of Wisonsin prep. MAD/PH/755, April 1993.

[6] P. Langacker and N. Polonsky, Phys. Rev. D47 (1993) 4028; U. of Pennsylvania preprint, UPR-0556T, May 1993.

[7] M. Carena, S. Pokorski and C.E.M. Wagner, Nucl. Phys. B406 (1993) 59.

[8] H. Arason, D. J. Castaño, B. Keszthelyi, S. Mikaelian, E. J. Piard, P. Ramond and B. D. Wright, Phys. Rev. Lett. 67 (1991), 2933.

[9] S. Kelley, J.L. Lopez and D.V. Nanopoulos, Phys. Lett. B278 (1992) 140.

[10] S. Dimopoulos, L. Hall and S. Raby, Phys. Rev. Lett. 68 (1992) 1984, Phys. Rev. D45 (1992) 4192. Phys. Rev. D 45 (1992).

[11] K. Hikasa et al, Particle Data Group, Phys. Rev. D 45 (1992).

[12] H. Georgi, H. Quinn and S. Weinberg, Phys. Rev. Lett. 33 (1974) 451.

S. Dimopoulos and H. Georgi, Nucl. Phys. B193 (1981) 150.

N. Sakai, Z. Phys. C11, 153 (1981)

E. Witten, Nucl. Phys. B188, 513 (1981).

[13] J. Ellis, S. Kelley and D.V. Nanopoulos, Phys. Lett. B260 (1991) 131;

P. Langacker and M.X. Luo, Phys. Rev. D44 (1991) 817;

F. Anselmo, L. Cifarelli, A. Peterman and A. Zichichi, Nuovo Cimento 104A (1991) 1817 ;

U. Amaldi, W. de Boer and H. Fúrstenau, Phys. Lett. B260 (1991) 447. 
[14] W. A. Bardeen, A. J. Buras, D. W. Duke and T. Muta, Phys. Rev. D 18 (1978) 3998.

[15] J. Ellis, S. Kelley and D. V. Nanopoulos, Phys. Lett. B249 (1990) 441; Phys. Lett. B287 (1992) 95; Nucl. Phys. B373 (1992) 55;

F. Anselmo, L. Cifarelli, A. Peterman and A. Zichichi, Nuovo Cimento 105A (1992) 581 ;

R. Barbieri and L. Hall, Phys. Rev. Lett. 68 (1992) 752;

J. Hisano, H. Murayama and T. Yanagida, Phys. Rev. Lett. 69 (1992) 1014.

[16] G. G. Ross and R. G. Roberts, Nucl. Phys. B377 (1992) 571.

[17] R. Hempfling, DESY Preprint, DESY-93-092, July 1993.

[18] L.J. Hall, R. Rattazzi, U. Sarid, LBL preprint, LBL-33997, June 1993.

[19] M. Olechowski and S. Pokorski, Nucl. Phys. B404 (1993) 590.

[20] G. Altarelli, talk presented at the EPS Meeting, Marseille, July 1993.

[21] J. Sola, in Phenomenological Aspects of Supersymmetry, pg. 187, Springer - Verlag, 1992.

[22] G. Altarelli, R. Barbieri and F. Caravaglios, CERN Preprint, CERN-TH 6902/93. 